\title{
Design of a cartographic eye tracking-EEG user experiment
}

\author{
Merve Keskin ${ }^{\mathrm{a}, \mathrm{b}}$ *, Kristien Ooms ${ }^{\mathrm{a}}$, Philippe De Maeyer ${ }^{\mathrm{a}}$, Ahmet Ozgur Dogru ${ }^{\mathrm{b}}$ \\ ${ }^{a}$ Ghent University,merve.keskin@ugent.be,kristien.ooms@ugent.be,philippe.demaeyer@ugent.be \\ ${ }^{b}$ Istanbul Technical University,ozgur.dogru@itu.edu.tr \\ * Corresponding author
}

Keywords: eye tracking, EEG, user experiment, experimental design, spatial memory task

\begin{abstract}
:
This paper focuses on the design of a cartographic user experiment that employs both eye tracking (ET) and electroencephalogram (EEG). When creating such an experiment, one is confronted with a large number of (often conflicting) challenges which need to be resolved: quality of the recorded signals, design of appropriate stimuli and tasks, synchronization of the data, etc. The goal of the experiment is to explore the (cognitive) strategies of expert and novice map users through cognitive load measurements when they are asked to memorize and then remember a (part of) map content with varying levels of complexity. Because the procedure of memorizing a map content in order to retrieve it stimulates the cognitive map production in map users' brains and hence it causes a cognitive load which can be measured with ET and EEG techniques. Throughout the paper, we will address the design issues by emphasizing the content of the stimuli and task, procedures of how the experiment will be executed and psychological measures to indicate cognitive load. For this, we combined a within and between subjects design: two different groups of participants studied different groups of stimuli (with varying levels of complexity).
\end{abstract}

In order to organize this in a structured way, the experiment is composed of seven blocks containing 50 trials. These blocks are shown in a random order. Each block is related to a certain level of complexity, represented by a (group of) map feature classes that should be remembered: (i) the whole sketch map, (ii) roads and hydrography, (iii) roads and green areas, (iv) green areas and hydrography, (v) green areas, (vi) hydrography, or (vii) roads. As such the cognitive load demand of tasks in each block is different, because each block is dedicated to the retrieval of a different map feature class or a combination of classes.

Each trial in the experiment is composed out of two parts. First, a map stimulus to study, which is derived from Google maps at zoom level 15 with $1 \mathrm{~km}$ scale bar, is shown for seven seconds long (Figure 1). This zoom level was chosen considering the size of the display screen and the consistency of the cartographic generalization for this level of detail among all the stimuli included in the experiment. This part corresponds to a free-viewing condition in which participants were asked to study a map stimulus, focusing on certain main structuring elements of the map. Second, a response screen appeared (Figure 2) with four graphical response panel: sketch maps which reflects the map content relevant to the task and are prepared by digitizing the main structuring elements considering cartographic generalization principles. Only one of the options corresponds to the map stimulus that was shown. Participants were required to keep the correct answers in their memory $(\mathrm{a}, \mathrm{b}, \mathrm{c}, \mathrm{d})$ and indicate it in the next screen.

We recorded EEG and ET simultaneously throughout the experiment. Typically, a high number of trials is included in an EEG experiment to be able to filter out the noise in the EEG data because besides brain-related activity, EEG data consists of noise elicited from different external sources (e.g. muscle or blink artifacts, power line noise generated by electrical devices in the room). When the number of trials are increased, the chances of obtaining artifact-free trials are higher.

It is important to decide which ET and EEG metrics can be used to calculate the cognitive load. On the one hand, the cognitive load can be measured using EEG activity power spectrum. For instance, spectral power change across frontal and temporal channel locations under alpha frequency band is a good indicator of cognitive load. Therefore, we can average the alpha power $(8-13 \mathrm{~Hz})$ for 7 seconds-long EEG recordings (i.e. same as the duration of the stimulus on the screen) of all 50 trials for each block. Average alpha power can be computed for expert and novices groups separately to study the differences between them and also based on blocks to study the influence of cognitive load into recalling performance. On the other hand, ET data can be used to identify blinks, fixations and saccades within the EEG data for noise reduction. After preprocessing, eye metrics such as number of fixations and fixation durations for each trial can be calculated and correlated to EEG data for overall cognitive load estimation.

In short, this experiment design allows us to examine how cognitive load affects the recalling performance, and whether some features are recalled independently of task difficulty. If so, we can identify which features are recalled easily/primarily, especially when the task demands higher cognitive load. Therefore, this outcome can contribute to create cartographic products in a more effective way by indicating the potential benefits of implementing EEG in cartographic usability research. 
Google Maps

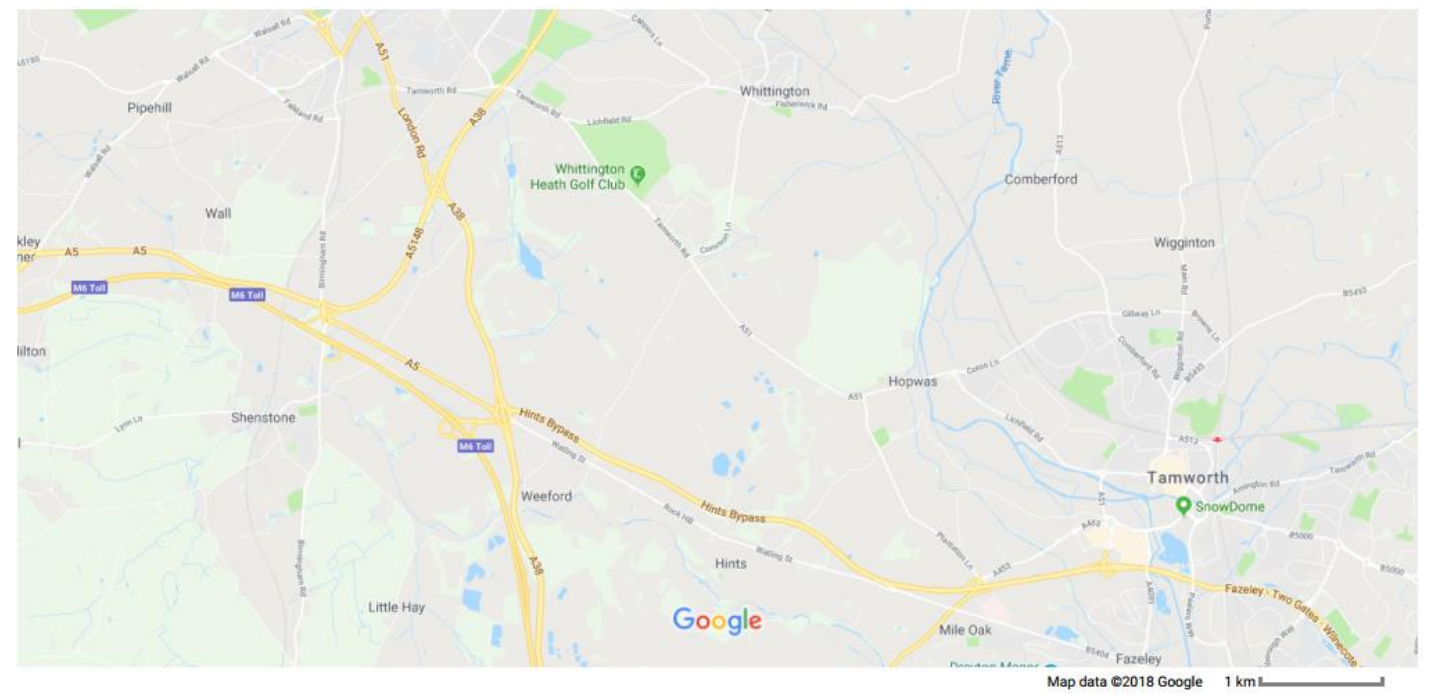

Figure 1. A map stimulus to study
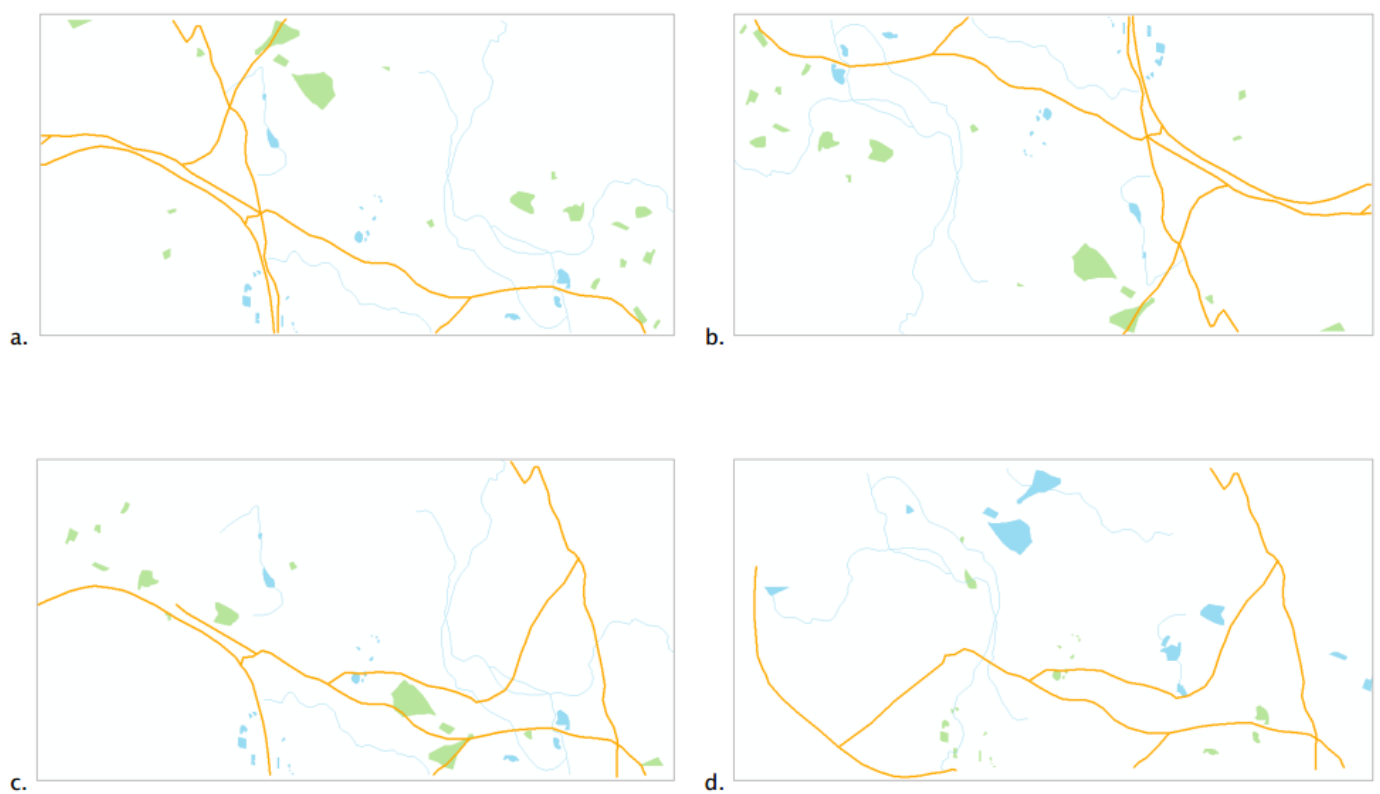

Figure 2. A graphical response screen with sketch maps (in this case for Block 1). 